\title{
Optical characterisation of photonic wire and photonic crystal waveguides fabricated using nanoimprint lithography
}

Borel, Peter Ingo; Frandsen, Lars Hagedorn; Lavrinenko, Andrei; Olsen, Brian Bilenberg; Nielsen, Theodor; Kristensen, Anders; Jensen, Jakob Søndergaard; Sigmund, Ole

\section{Published in:}

Proceedings European Conference on Optical Communication

Link to article, DOI:

10.1109/ECOC.2006.4801135

Publication date:

2006

Document Version

Publisher's PDF, also known as Version of record

Link back to DTU Orbit

Citation (APA):

Borel, P. I., Frandsen, L. H., Lavrinenko, A., Olsen, B. B., Nielsen, T., Kristensen, A., Jensen, J. S., \& Sigmund, O. (2006). Optical characterisation of photonic wire and photonic crystal waveguides fabricated using nanoimprint lithography. In Proceedings European Conference on Optical Communication (Vol. CD-ROM, pp. We1.2.4). IEEE. https://doi.org/10.1109/ECOC.2006.4801135

\section{General rights}

Copyright and moral rights for the publications made accessible in the public portal are retained by the authors and/or other copyright owners and it is a condition of accessing publications that users recognise and abide by the legal requirements associated with these rights.

- Users may download and print one copy of any publication from the public portal for the purpose of private study or research.

- You may not further distribute the material or use it for any profit-making activity or commercial gain

- You may freely distribute the URL identifying the publication in the public portal 


\title{
Optical characterisation of photonic wire and photonic crystal waveguides fabricated using nanoimprint lithography
}

Peter I. Borel (1), Lars H. Frandsen (1), Jacob Fage-Pedersen (1), Andrei V. Lavrinenko (1), Brian Bilenberg (2),

Theodor Nielsen (2), Anders Kristensen (2), Jakob S. Jensen (3), and Ole Sigmund (3)

1 : COM·DTU, Department of Communications, Optics and Materials, Nano•DTU,

Technical University of Denmark, DK-2800 Kgs Lyngby, Denmark. pib@com.dtu.dk

2 : MIC - Department of Micro and Nanotechnology, Nano•DTU, Technical University of Denmark

3 : MEK, Department of Mechanical Engineering, Nano•DTU, Technical University of Denmark

\begin{abstract}
We have characterised photonic-crystal and photonic-wire waveguides fabricated by thermal nanoimprint lithography. The structures, with feature sizes down below $20 \mathrm{~nm}$, are benchmarked against similar structures defined by direct electron beam lithography.
\end{abstract}

\section{Introduction}

The development of silicon-based nanophotonic structures such as photonic wires and 2D photonic crystal waveguides ( $\mathrm{PhCWs}$ ) has been an immensely active research area during the last few years. Today, the research has matured to a level where it is possible to fabricate low-loss $2 \mathrm{D}$ photonic crystal waveguides using electron beam lithography (EBL) [1] and deep UV lithography (DUVL) [2]. EBL, in particular, provides nanophotonic structures with extremely high resolution, and this fabrication method is appropriate for many research investigations. However, being a serial fabrication process it is not optimal for mass fabrication of photonic devices. DUVL, on the other hand, is ideally suited for mass fabrication. In this case, however, the production volume must be large enough to justify the immensely large costs affiliated with the fabrication method. Another disadvantage utilising DUVL is that the fabrication tolerances presently are pushed to their limits to obtain acceptable structures, leaving only little room for improvement.

Fabrication of nanophotonic structures using nanoimprint lithography (NIL) [3] is emerging as a cheap alternative that offers parallel component production in combination with nanometer scale resolution comparable to EBL. Here, we report first results on the optical performance of nanophotonic structures fabricated using NIL. We find that the structures fabricated with NIL have transmission features very similar to structures fabricated directly with EBL.

\section{Fabrication using nanoimprint lithography}

The silicon stamp used in the imprint process is fabricated by EBL (JEOL JBX9300FS) utilising a negative resist film of TEBN-1 (Tokuyama Corp., Tokyo, Japan) with a thickness of $50 \mathrm{~nm}$. The written structures are transferred $100 \mathrm{~nm}$ into the silicon substrate by a highly anisotropic reactive ion etch. After the silicon etch the remaining resist is removed using an oxygen plasma and an anti sticking layer from a $\mathrm{C}_{4} \mathrm{~F}_{8}$ plasma is deposited.

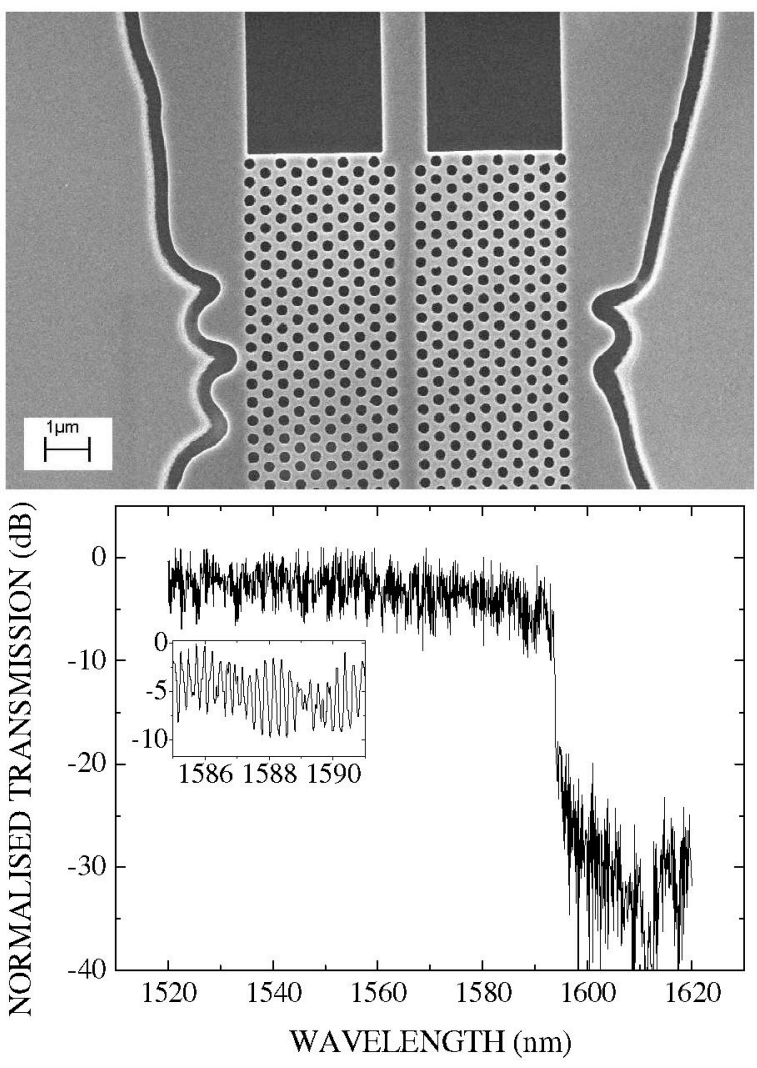

Figure 1: (top) SEM image of a photonic wire adjacent to a 10- $\mu \mathrm{m}$ long W1 PhCW fabricated in SOl by NIL. The etch patterns seen on the outer sides are caused by the controlled flow of excess polymer during the imprint process. (bottom) Measured transmission spectrum for quasi TE polarised laser light through the structure. Inset shows a zoom-in on the spectrum.

An 80-nm thick film of $\mathrm{mr}-\mathrm{I}$ T85 nanoimprint resist (micro resist technology $\mathrm{GmbH}$, Berlin, Germany) is spincoated onto a silicon-on-insulator (SOI) and the stamp is imprinted herein using an EVG imprint tool. The imprint parameters result in a complete filling situation of the stamp in the nanostructured areas, resulting in 100-nm deep holes in the resist. The nanoimprinted patterns are transferred into the $320-\mathrm{nm}$ thick top silicon layer of the SOI substrate by using an optimised $\mathrm{SF}_{6}$-based inductively coupled 
plasma etch. The etch selectivity of silicon over the resist allows for pattern transfer of the imprinted holes through the full thickness of the device silicon layer of the SOl substrate.

\section{Optical characterisation and results}

The fabricated structures have been characterised optically by transmission measurements using quasi transverse electric (TE) polarised light from a laser source in the wavelength region from $1520-1620 \mathrm{~nm}$ and broadband LED diodes covering 1360-1620 nm.

Figure 1 shows a W1 PhCW (i.e., a waveguide where the defect is formed by removing one row of holes in the $\Gamma-\mathrm{K}$ direction), fabricated by NIL, and the corresponding measured transmission spectrum. The ripples in the spectrum are due to Fabry-Pérot oscillations caused by reflections from the end facets of the sample as seen from the inset. The sharp cutoff (in this case around $1590 \mathrm{~nm}$ ) and the high and uniform transmission level below the cut-off wavelength are clear footprints of a successfully fabricated $\mathrm{PhCW}$. The spectrum and the transmission level (including both the photonic wire and $\mathrm{PhCW}$ parts) are found to be comparable to those of samples fabricated with EBL [4].

Recently, we have proposed a novel inverse design strategy called topology optimisation (TO) [5], which allows for designing nanophotonic structures with enhanced functionalities. In some cases this inverse design method proposes optimised designs with feature sizes down to $\sim 20 \mathrm{~nm}$. Hence, such structures are very challenging to fabricate even with $\mathrm{EBL}$ and will serve as excellent benchmarks for how well NIL fabricated structures perform.

Figure 2 shows a PhCW wavelength-selective splitter designed using TO so that the shorter wavelengths primarily are transmitted through one output arm and the longer wavelengths through the other. The figure shows the originally designed structure and its transmission performance, calculated using a 3D finite-difference time-domain (FDTD) scheme [4]. Also shown is a SEM image of the fabricated structure and the measured transmission spectra. It is seen that the fabricated structure closely resembles the original design and likewise that the transmission performance of the fabricated structure and the original design are very similar both with respect to the transmission level and the wavelength dependent features. Hence, the NIL fabrication of the challenging nanophotonic TO design has been successful. In addition, it is seen that the TO compact wavelength splitter functions as designed, namely as a fairly efficient high pass/low pass wavelength filter.
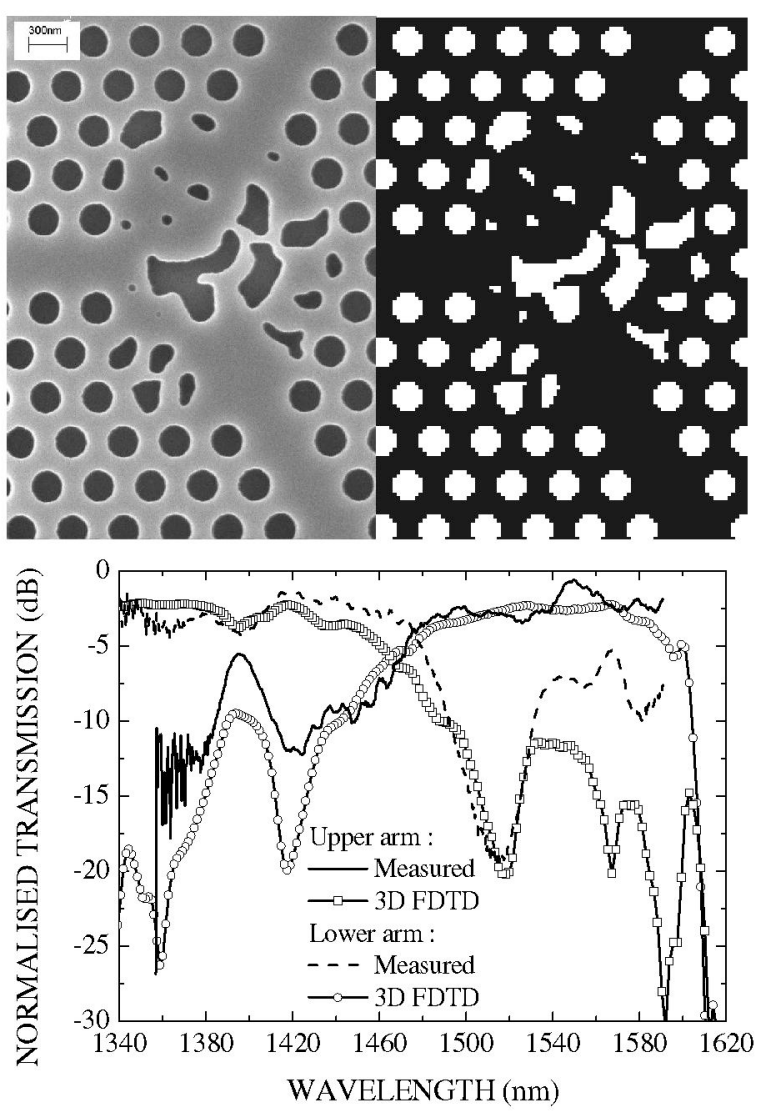

Figure 2: (top, left) SEM image of a wavelengthselective splitter designed using TO and fabricated in SOI by NIL. (top, right) The original TO design. Light enters the component from the left side. (bottom) Normalised measured transmission below the cut-off wavelength for quasi TE polarised light from the two output arms. Also shown are $3 D$ FDTD calculations for the transmission through the output arms of the originally designed structure. The $3 D$ FDTD calculations have been blue shifted by $0.5 \%$ in wavelength for clarity.

\section{Conclusions}

We have used NIL to fabricate SOl based nanophotonic structures with feature sizes down below $20 \mathrm{~nm}$. The NIL fabricated devices perform comparably to the direct $E B L$ defined devices and the obtained results are in good agreement with $3 \mathrm{D}$ FDTD calculations. Thus, we have demonstrated the feasibility of NIL as a cost efficient fabrication technique for silicon-based nanophotonics.

\section{References}

1 E. Kuramochi et al. Phys. Rev. B, 72 (2005), $161318(\mathrm{R})$

2 M. Settle et al. Opt. Express, 14 (2006), 2440

3 S. Chou et al. Appl. Phys. Lett., 67 (1995), 3114

4 A. Lavrinenko et al. Opt. Express, 12 (2004), 234

5 P.I. Borel, et al. Opt. Express, 12 (2004) 1996 